\title{
901 BACILLUS CALMETTE-GUERIN CAN SUBVERT PATIENTS ANTITUMOR IMMUNE RESPONSE BY DOWNREGULATING HLA-I EXPRESSION ON CANCER CELLS
}

'Mathieu Rouanne*, ${ }^{2} J u l i e n$ Adam, ${ }^{1}$ Camelia Radulescu, ${ }^{1}$ Diane Letourneur, ${ }^{1}$ Severine Mouraud, ${ }^{1}$ Delphine Bredel, ${ }^{1}$ Anne-Gaelle Goubet, ${ }^{3}$ Tuan Zea Tan, ${ }^{1}$ Amelie Bigorgne, ${ }^{4}$ Michael Dussiot, 'Lambros Tselikas, 'Sandrine Susini, ${ }^{1}$ FrançoisXavier Danlos, ${ }^{1}$ Roman Chabanon, ${ }^{1}$ Nicolas Signolle, ${ }^{1}$ Anna Schneider, ${ }^{5}$ Sophie Vacher, ${ }^{5}$ Ivan Bieche, ${ }^{1}$ Thierry Lebret, ${ }^{1} Y$ ves Allory, ${ }^{1}$ Jean-Charles Soria, ${ }^{6}$ Jean Paul Thiery, 'Laurence Zitvogel, ${ }^{1}$ Aurelien Marabelle. 'Gustave Roussy, New York, NY, France; ${ }^{2}$ Hopital Paris Saint Joseph, Paris, France; ${ }^{3}$ Cancer Institute Singapore, Singapore, Singapore; ${ }^{4}$ Institut Imagine, Paris, France; ${ }^{5}$ Curie Institute, Paris, France; ${ }^{6}$ Singapore Cancer Institute, Singapore, Singapore

Background Patients with high-risk non muscle-invasive bladder cancer (NMIBC) frequently relapse after standard BCG immunotherapy and have a dismal outcome after progression to muscle-invasive bladder cancer (MIBC). ${ }^{12}$ The mechanisms of tumor resistance to such immunotherapy remain elusive.

Methods We performed functional assays of fresh human bladder tumors mixed with BCG, reinforced with in vitro experiments and in situ transcriptomics analyses together with immune profiling by immunohistochemistry (IHC) in a cohort of T1 NMIBC pre- and post BCG therapy.

Results We found two distinct patterns of BCG-induced immune subversion. In the first pattern, intracellular infection by live BCG was associated with HLA-I loss and epithelial-tomesenchymal transition characteristics. Mechanistically, LC3GFP reporter cell line showed a significant induction of autophagy upon BCG exposure. HLA-I deficient tumors displayed a myeloid immunosuppressive microenvironment together with an upregulation of autophagy-related genes, and dismal outcome. Conversely, HLA-I+ BCG-treated tumors generated a Th1 type of immune response associated with an upregulation of exhaustion markers. Such patients had a very favorable outcome upon radical surgery.

Conclusions We surmise that HLA-I expression in bladder cancers does not result from immunoediting but rather from HLA-I molecules endocytosis related to autophagy induction in infected cancer cells. Cancer cells HLA-I scoring by immunohistochemistry staining can be easily implemented by pathologists in routine practice to stratify future bladder cancer patient treatment strategies.

\section{REFERENCES}

1. Pietzak EJ, Zabor EC, Bagrodia A, et al. Genomic differences between "primary" and "secondary" muscle-invasive bladder cancer as a basis for disparate outcomes to cisplatin-based neoadjuvant chemotherapy. Eur Urol 2019;75(2):231239.

2.

Patrick J Hensley, Kelly K Bree, Matthew T Campbell, et al. Progression of disease after BCG therapy: refining patient selection for neoadjuvant chemotherapy before radical cystectomy. J Urol 2021 June 29;101097JU0000000000001943.

Ethics Approval Our study obtained ethics approval from the Foch Hospital Ethics Committee (IRB00012437). All the participants gave informed consent before taking part.

Consent We surmise that HLA-I expression in bladder cancers does not result from immunoediting but rather from HLA-I molecules endocytosis related to autophagy induction in infected cancer cells. Cancer cells HLA-I scoring by immunohistochemistry staining can be easily implemented by pathologists in routine practice to stratify future bladder cancer patient treatment strategies. 